\title{
Criticality and Punctuated Equilibrium in a Spin System Model of a Financial Market
}

\author{
A. PONZI and Y. AIZAWA \\ Department of Applied Physics, School of Science and Engineering, Waseda University, \\ Shinjuku, Tokyo 169-8555, Japan
}

\begin{abstract}
We describe a financial market model which shows a non-equilibrium phase transition.

Near the transition punctuated equilibrium behaviour is seen, with avalanches occuring on all scales. This scaling is described by an exponent very near 1 . This system shows intermittent time development with bursts of global synchronization reminiscent of a market rollercoaster.
\end{abstract}

\section{Introduction}

Recently much attention has been given to connections between criticality and selforganized criticality (SOC) and evolutionary phenomenon, particularly punctuated equilibrium, and to connections between SOC and synchronization. We describe a model which we hope draws some connection between these 2 ideas. SOC has been proposed to describe out of equilibrium systems that are critical, that self-organize into a scale invariant critical state without tuning of a control parameter and show fractal time series. [1] Evolution SOC type models [15, 16, 17] have been proposed to explain punctuated equilibrium. [18, 19] Punctuated equilibrium is the phenomenon observed in the fossil record where long periods of stasis are interrupted by sudden bursts of evolutionary change. 
Kaufman and Johnsen [14 have modeled co-evolution, where agents live on a coupled fitness landscape and walk around by random mutation, only moves to higher fitnesses are allowed. Once at a local maximum the walk stops until moves by another agent deform the lanscape so the agent is no longer at a maximum. Kauffman [14 has linked this to SOC. Bak, Sneppen and Flyvbjerg [15, 16] have taken a similar approach. They define a species as a barrier to increasing fitness and choose the least fit, then randomly change its barrier and the barriers of other agents. The system evolves to a critical state with a selforganized fitness threshold. SOC has also been linked to periodic behaviour. [10, 11, 12, 13] A.Corral et al and Bottani [11, 10], say there is a close relationship between SOC and synchronization. SOC appears when a system is perturbed which otherwise should synchronise totally or partially. The perturbation may be open boundary conditions rather than periodic [10, 13] or it may be randomness present in initial conditions which is preserved by the dynamic, or it may be the addition of noise. The model we study appears to link these 2 ideas with the emergence of avalanches of partial synchronization on all scales. However all these models are real space models whereas ours is a mean-field model with no spatial dimension. Our model is entirely determistic, the critical state is produced by certain initial conditions, indeed other initial conditions produce completely periodic states. Our model also is not strictly speaking an SOC model since critical behaviour only occurs for a certain range of the parameter and then only for certain initial conditions. A complete analysis of the intial conditions is outside the scope of this paper.

Our model was originally motivated as a model of the behaviour of speculating traders 
in a financial market in the spirit of co-evolution. Recent results have shown stock price time series to be fractal with Hurst exponent different from 0.5, [21] and with positive lyapunov exponent. [2, 3, 4, Scrambling daily returns changes the Hurst exponent back to 0.5. Large crashes have been supposed to be due to exogenous shocks, where information enters the market randomly. However large crashes interspersed with periods of slow growth are strongly reminiscent of punctuated equilibrium. Indeed Mandlebrot has noted large changes of cotton prices occur in oscillatory groups and the movement in tranquil periods is smoother than predicted. [22] Scaling behavior has been noted in a financial index and in the size of companies. [25, 26] Stanley et al have noted 'scaling laws used to describe complex systems comprised of many interacting inanimate particles (as in many

physical systems) may be usefully extended to describe complex systems comprised of many interacting animate subsystems (as in economics).'

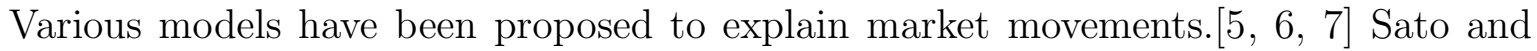
Takayasu have proposed a threshold type model. [8, 9] Since critical states can produce avalanches on all scales, without the need for exogenous shocks, we believe critical type dynamics are present in financial market dynamics.

\section{Model}

We hope to model co-evolutionary phenomenon where the micro-level itself defines the macro-level but is also slaved to the macro-level. This is very evident in speculative financial market dynamics where a collection of individuals (micro) trade therby creating 
a price time series (macro), but determine their trading behaviour by reference to this same price series and other macro variables. We desired to make a model in analogy to this phenomenon.

This is a highly stylized toy model of a stock-market.There are $N$ agents which are represented by spins $s_{i}(t)$, where $s_{i}(t)=1$, means the agent $i$ owns the stock and $s_{i}(t)=$ -1 means doesn't own the stock at time t. Each agent also has an absolute fitness $F_{i}(t)$ and a relative fitnes $f_{i}(t)=F_{i}(t)-F(t)$ where the mean-fitness $F(t)=\frac{1}{N} \sum_{i=1}^{N} F_{i}(t)$. We believe speculative traders are part of 2 crowds, bulls and bears, and our macrovariable is 'groupthink' $G(t)$, defined by,

$$
G(t)=\Delta P(t)=\frac{1}{N} \sum_{i=1}^{N} s_{i}(t)
$$

The dynamic is:

$$
\begin{gathered}
\Delta s_{i}(t)=s_{i}(t+1)-s_{i}(t)= \begin{cases}-2 s_{i}(t) & f_{i}(t) \leq 0 \\
0 & f_{i}(t)>0\end{cases} \\
\Delta F_{i}(t)=F_{i}(t+1)-F_{i}(t)=-\frac{1}{2} \Delta s_{i}(t) G(t)+\frac{1}{2}\left|\Delta s_{i}(t)\right| c
\end{gathered}
$$

The dynamic is synchronous and deterministic. First $G(t)$ and $F(t)$ are calculated then all agents are updated according to (2) and (3). The price $P(t)$ is defined by (1) and $P(0)=0$. Initially $s_{i}(0)$ are chosen randomly with probablity $1 / 2$ and $F_{i}(0)$ are chosen randomly from the interval $[-1,1]$.

$G(t)$ measures the bullishness or bearishness of the crowd. Although different to ours Callan and Shapiro mention groupthink in Theory of Social Imitation 23] and Vaga's 24, 21] Coherent Market Hypothesis explicitly includes a variable called groupthink. 
We believe speculative agents determine their spin state dependent on whether they believe the market will move in their favour in the future. Therefore our agents have an absolute fitness $F_{i}(t)$ which measures their perception of whether they are in a good position with respect to the future. If $F_{i}(t)$ is relatively high their state will be stable and if $F_{i}(t)$ is relatively low they will want to change their current state. Many ways to define $F_{i}(t)$ are possible. In this model we define it by analogy to Plummer. Agents consider the market to be 'overbought' or 'oversold'. In our simplistic model this is measured by $G(t)$. An agent is fit if it is in the minority group. According to Plummer when most agents are in one position then there must be less buying into this position (because there are only a finite amount of agents) and therefore the market will eventually correct itself (change direction) because its growth will not be sustainable. It is always profitable then to be in the minority group before a correction. At a correction the dominant crowd breaks, the macro position dissolves, the market may crash, and subsequently bull and bear crowds will begin to reform. In fact at these times the agents may trade in 2 macro-clusters or chaotically with the market attaining high volatility which persists for some time. This type of trader has been called a sheep trader [5, 6, in contrast to fundamentalists speculators and non-speculators. Therefore in this model an agents fitness is increased if it changes from the majority group to the minority group, with the increase proportional to the size of the majority. The opposite is applied if it changes the other way. If an agent doesn't change its state then it's absolute fitness $F_{i}(t)$ is not changed, regardless of whether $G(t)$ changes. An agent also has a relative fitness $f_{i}(t)$. The $f_{i}(t)$ are the 
behaviour controlling variables in this model. They may change in 2 ways. Firstly an agent $\alpha$ may change its state $s_{\alpha}(t)$ thereby directly changing $F_{\alpha}(t)$ and $f_{\alpha}(t)$. This is similar to a single adaptive move on a fitness landscape by an individual optimizing agent. Secondly co-evolution may occur. Here an agent's relative fitness $f_{\beta}(t)$ may change due to changes in the other agents fitnesses $F_{i}(t)$ changing $F(t)$ while $F_{\beta}(t)$ remains constant.

To model evolution then we follow natural selection by analogy and mutate unfit agents and leave fit agents unchanged (although their relative fitnesses may change). as in Kaufman, [14] and Bak et al [15, 16]. Mutation is considered to be a state change and this changes an agent's fitness according to (3). In this model since their are only 2 possible states this means we simply flip state. (In a more extensive model this would correspond to changes to an ownership portfolio vector). To decide which spins flip we could compare pairs of fitnesses and change the least fit. That is we could choose 2 agents $\alpha$ and $\beta$ and let them compete so that $F_{\alpha}>F_{\beta}$ then we say $s_{\alpha}(t+1)=s_{\alpha}(t)$ and $s_{\beta}(t+1)=-s_{\beta}(t)$. However in this paper we simply take a mean-fitness approach. That is all agents $i$ whose fitnesses $F_{i}(t)$ fulfill $F_{i}(t) \leq F(t)$ ie $f_{i}(t) \leq 0$ flip there spins and their fitnesses change according to (3). All other agent's states and absolute fitnesses $F_{i}(t)$ do not change although their relative fitnesses $f_{i}(t)$ of course do. Therefore fit agents which could be considered to be at a local maximum do not change their states until the mean-fitness $F(t)$ has become equal to their fitnesses $F_{i}(t)$.

This means the fitnesses are all internally defined emergent properties as in co-evolution. Of course if there is no overall crowd polarisation then changing state does not change 
fitness.

Therefore the fitness update rule (3) can be seen as the adaptive walk part and this is the reason why we do not simply set $F_{i}(t)=-s_{i}(t) G(t)$ or $\Delta F_{i}(t)=-s_{i}(t) G(t)$ continuously for all agents. We hope agents will take time to walk out of unfit states and that fit maxima will be created which persist for some time. Our absolute fitness is therefore cumulative and is only changed for unfit agents. More realistically we could think of agents imperfectly sampling the market ie $G(t)$ at a series of times to determine their current absolute fitness. In fact we see that the concept of relative fitness and absolute fitness are very similar to the concept of 'bounded rationality.' An agents rationality is bounded because he only makes local adaptive moves and can percieve only his absolute fitness but not the overall mean-fitness or his relative fitness

This fitness of the position is natural in the sense that it can be seen as a kind of potential for future profit, usually termed 'utility' in economics. The fitter an agent is the less likely it will want to change its state, the more stable it is, because it believes the market to be oversold in its favour.

Since $G(t)$ will on average be 0 , in addition in equation (3) we include a very small control parameter $c$ which controls the driving rate. We add this to all fitnesses below the mean so that unfit agents on average will be come fitter and interact with the fit agents. This is a general characteristic of evolutionary systems that single entity moves on fitness landscape should be on average uphill.

Our market price $P(t)$ is defined by $\Delta P(t)=G(t)$, ie price increases while more people 
own the share than don't own it, and the price is theoretically unbounded as it should be. Positive groupthink means positive increase and vice-versa. This is similar to the way prices are usually defined by $\Delta p(t) \propto Z(t)$, where $Z(t)$ is the excess demand for something. This model does not included a fixed amount of shares. Indeed any trader may independently buy or sell a share without the notion of swapping. This reflects the fact that this is a model of only speculative behaviour, and part of a much larger of pool of shares. However a more realistic model should include a fixed amount of shares.

This model is intended to be a suggestive illustration rather than a realistic stockmarket.

\section{Results}

Shown in Fig.1a is a time series for the fitnesses $F_{i}(t)$ for an $N=80$ system for $c=0.01$. Punctuated equilibrium behaviour is clearly visible, with periods of relative stasis interspersed with sudden jumps. Although not shown the mean-fitness time series $F(t)$ shows changes on all scales similar to a devils staircase. Also shown in Fig.1b is the corresponding daily returns time-series $\Delta P(t)=G(t)$, this also shows calm periods and sudden bursts of high volatility. Infact this behaviour is a kind of intermittent partial synchronization. Shown in Fig.2 is the same time series but with a small portion magnified. Macroscopic synchronization can be seen. Partial synchronizations show various different periods and complexities, and persist for various lengths of time. Clustering allows synchronized spins to trade in phase with groupthink $G(t)$ thereby rapidly increasing 
there fitnesses, or out of phase thereby becoming less fit, this is the origin of the sudden large changes in fitness. Also at these times the fitness deviation suddenly increases, (not shown). Between periods of large-scale partial synchronization with high volatility, periods of calm are characterized by a small even number of spins flipping in anti-phase, they therefore increase their $F_{i}$ only slowly due to the driving parameter c, the returns $G(t)$ remaining roughly constant during these periods with the fitness deviation decreasing. (Of course anti-phase flipping with no average increase in fitness is prevented in a real market by a fixed transaction cost. A more realistic model must include this.) When the mean-fitness $F(t)$ which is usually increasing crosses some non-flipping $F_{i}$, this spin flips and may cause the $F(t)$ to cross some more $F_{i}$, possibly starting an avalanche. This only happens when the total fitness deviation is small.

Shown in Fig. 3 are two price $P(t)$ time series for $c=0.013$. Their fractal slightly repetetive pattern is highly reminiscent of real financial time series.

Since this model is deterministic, completely periodic states are also possible. Shown in Fig.4 is the average of the quantity $<R(t)>$ where $R(t)=\sum_{i=1}^{N}\left|\Delta s_{i}(t)\right|$ is the amount of spins which flip at any time and $\langle\ldots\rangle$ denotes time averaging. In Fig.4a are time series for $c=0.0113$ while Fig. $4 \mathrm{~b}$ is $c=0.01$.

Fig.4a shows one time series finds the 2 cluster periodic state, where 2 groups alternately topple. Here 59 time series are included in the non-periodic state. If this is a transient it is super long even for the moderate size $N=200$. Fig.4b shows at more regular $c$ values the system can find periodic states with larger amounts of clusters. Roughly 
half of the 60 series investigated become periodic by $t=2.5 \times 10^{7}$. Shown in Fig.5a is $\left\langle R(t)>\right.$ plotted against $c$. Infact to construct this plot we discarded $3 \times 10^{7}$ time steps and then averaged over the next 20,000, each point represents a different initial condition and there are 8 for each cost $c=0.001 x+0.000138, x$ is an integer. For small cost critical type behaviour is evident with a sudden phase transition at $c=0$. This is of course because at negative $c$ less fit spins continuously flip and never interact with spins at greater than mean fitness. In fact the system divides into a frozen solid fit component and an unfit gaseous type component for negative $c$. The size of the frozen component depends on the initial conditions as can be seen from the points at negative cost. For larger positive cost an upper branch of periodic attractors at $<R(t)>=100$, half the system size, is evident, the system has settled into 2 alternately toppling clusters which interleave the mean-fitness $F(t)$. The lower branch is characterised by the punctuated equilibrium state shown in Fig.1. Fig.5b shows the time average $<S(t)>$ of an entropy type quantity of the fitness distribution $S(t)$ given by, $S(t)=-\sum_{i=1}^{N} \frac{\left|f_{i}(t)\right|}{f(t)} \ln \frac{\left|f_{i}(t)\right|}{f(t)}$ where $f_{i}(t)$ is the fitness deviation and $f(t)=\sum_{i=1}^{N}\left|f_{i}(t)\right|$. The averaging is the same as for $<R(t)>$. For this $N=200$ system the maximum $S=\ln 200 \approx 5.3$ and the periodic points at positive and negative cost are very near this. The punctuated equilibrium state which exists near the transition is more ordered at lower entropy.

This is our first evidence of critical behaviour for small $c$. Second evidence is obtained by looking at the distribution of avalanches. In the punctuated equilibrium state the system finds a state characterised by fluctuations on all scales. Shown in Fig.6 is the 
distribution of $P(R)$ against $R$ where $P(R)$ is the probability of an avalanche of size $R$. These are distributions of avalanches for 1 time series for 3 different system sizes. They are not ensembles of time series, this distribution is independent of the initial conditions and any non-periodic time series contains all avalanche sizes.

The time series were of length $T=16,000,000$, near the transition point at $c=0.0113$. The distribution shows scale invariance, $P(R) \sim R^{\alpha}$ up to about half the system size. At half the system size there is a peak where the system almost finds the periodic attractor and spends more time in these states. After this the distribution continues to the cutoff near the system size. The scaling exponent $\alpha$ taken from the $N=3000$ distribution is $\alpha=-1.085 \pm 0.002$. Also shown in Fig.7 is the distribution of magnitude of changes in mean fitness $\Delta F(t)=|F(t+1)-F(t)|$, the steps in the devils staircase. The time series are the same as in Fig.6 for 2 different system sizes, there is no ensemble averaging. In fact the two distributions for $N=1500,923$ are almost identical, if we were to superpose them, only one could be seen. This is also true for other system sizes. Peaks appear at $\Delta F \approx 0.12,0.5,0.75$. Between the peaks we see scaling regimes. Here we see at least 2 scaling regimes, $P(\Delta F) \sim \Delta F^{\beta}$ where for $\Delta F \leq 0.1, \beta=-1.25 \pm 0.003$ and for $0.1 \leq \Delta F \leq 0.4, \beta=-1.39 \pm 0.02$. Possibly there is another scaling regime for $0.55 \leq \Delta F \leq 0.7$. 


\section{Conclusion}

This model illustrates an interesting relation between critical phenomenon and punctuated equilibrium on the one hand, and between partial synchronization and punctuated equilibrium on the other hand. The system synchronizes for certain cost $c$ and certain intial conditions, otherwise it shows critical behaviour, similar to the SOC models cited in the introduction. We believe this deserves further investigation. We also find an interesting phase transition.

Some typical behaviour of money markets is present here, especially the periods of low volatility, where the price is relatively stable and the fitness grows slowly while the fitness deviation decreases slowly, interrupted by shorter periods of persistent high volatility and macroscopic oscilations which are observed in real time series. We wonder if like in earthquake dynamics, which are often modelled by SOC dynamics, a large crash in a real financial market is preceded by some smaller self-reinforcing oscilatory pre-shock, as is seen in our dynamics here. Also the time price time series is highly suggestive of real time series, with formations similar to 'double tops' and 'rebounds' described in quantitative analysis, produced by the near periodic macro behaviour which can appear. The slightly repetetive self-similarity reminds us of financial time series.

Many possible models of financial market dynamics can be plausibly suggested, included many exhibiting threshold dynamics, since data concerning the micro behaviour of individual traders is not available. 


\section{References}

[1] P. Bak, C. Tang, and K. Weisenfeld, Phys. Rev. Lett. 59 381, (1988). Phys. Rev. A. $38364,(1988)$.

[2] R.H. Day and P. Chen,(eds.) Nonlinear Dynamics and Evolutionary Economics Oxford University Press. (1993)

[3] W.A. Barnett, A.P. Kirman, M. Salmon, (eds.) Nonlinear Dynamics and Economics, Proceedings of the Tenth International Symposium in Economic Theory and Econometrics Cambridge University Press (1996)

[4] P. Chen, Trends, shocks, persistent cycles in evolving economy: Business cycle measurement in time-frequency representation. in [3], K. Wen, Continuous-time chaos in stock market dynamics, in [3]

[5] P. Chen, Searching for Economic Chaos: A challenge to Econometric Practice and Nonlinear Tests [2], W. Huang and R.H. Day, Chaotically Switching Bear and Bull Markets: The Derivation of Stock Price Distributions from Behavioral Rules [2]

[6] E.C. Zeeman, On the unstable behaviour of stock exchanges Journal of Mathematical Economics 1 39-49. (1974)

[7] R.G. Palmer,W. Brian Arthur, J.H. Holland, B. LeBaron, Artificial economic life: a simple model of a stockmarket Physica D 75 (1994), 264-274.

[8] A. Sato, H. Takayasu, Physica A 250 (1998) 231-252. 
[9] H.Takayasu, H.Miura, T.Hirabayashi,K.Hamada, Statistical properties of deterministic threshold elements- the case of the market price. Physica A 184 (1992) 127-134.

[10] S. Bottani, Pulse-coupled Relaxation Osscilators: From Biological Synchronization to Self-Organized Criticality Phys.Rev.Lett. 74 21, 4189-4192, (1995).

[11] A. Corral, C.J. Perez, A.Diaz-Guilera and A.Arenas, Self-organized Criticality and Synchronisation in a lattice model of Integrate and Fire Oscillators Phys. Rev. Lett. 74 1, 118-121, (1995).

[12] A.A. Middleton and C. Tang, Self-organized criticality in Nonconserved Systems Phys. Rev. Lett. 74 5, 742-745, (1995).

[13] J.E.S.Socolar,G.Grinstein,C.Jayaprakash, On self-organized criticality in nonconserving systems Phys. Rev. E 47 4, 2366-2376, (1993).

[14] S.A.Kauffman and S.Johnsen, Co-evolution to the Edge of Chaos: Coupled Fitness Landscapes, Poised States and Co-evolutionary Avalanches J.Theor.Biol. 49 467$505,(1991)$

[15] P.Bak and K.Sneppen, Punctuated Equilibrium and Criticality in a Simple Model of Evolution Phys. Rev. Lett. 71 4, 4083-4086, (1993).

[16] H.Flyvbjerg, K.Sneppen, P.Bak, Mean field Theory for a Simple Model of Evolution Phys. Rev. Lett. 71 24, 4087-4090, (1993). 
[17] R.V. Sole and S.C. Manrubia, Criticality and unpredictability in macro-evolution Phys. Rev. E 55 4, 4500-4507. (1997)

[18] D.M.Raup, Biological Extinction in Earth History Science 231 1529-1533, (1986).

[19] S.J.Gould, N.Eldredge, Punctuated Equilibrium comes of age Nature 366 223, (1993).

[20] T.Plummer, Forecasting Financial Markets Kogan Page, London Ltd., (1989)

[21] E.E.Peters, Chaos and Order in the Capital Markets John Wiley \& Sons, Inc. (1996)

[22] E.E.Peters, 21] pp118-119.

[23] E.Callan and D.Shapiro, A Theory of Social Imitation Physics Today 27 July (1974)

[24] T.Vaga, The Coherent Market Hypothesis Financial Analysts Journal December (1991).

[25] M.H.R. Stanley, L.A.N Amaral, S.V. Buldyrev, S. Havlin, H. Leschhorn, P. Maass, M.A. Salinger, H. Eugene Stanley, Scaling Behaviour in the Growth of Companies Nature 379 804-806 (1996) Scaling Behavior in Economics: I. Empirical Results for Company Growth J.Phys. I France 7 (1997) 621-633. Scaling Behavior in Economics: II. Modeling of Company Growth. J.Phys. I France 7 (1997) 635-650.

[26] R.N.Mantegna, H. Eugene Stanley, Scaling Behaviour in the dynamics of an economic index. Nature 376 46-49 (1995) 

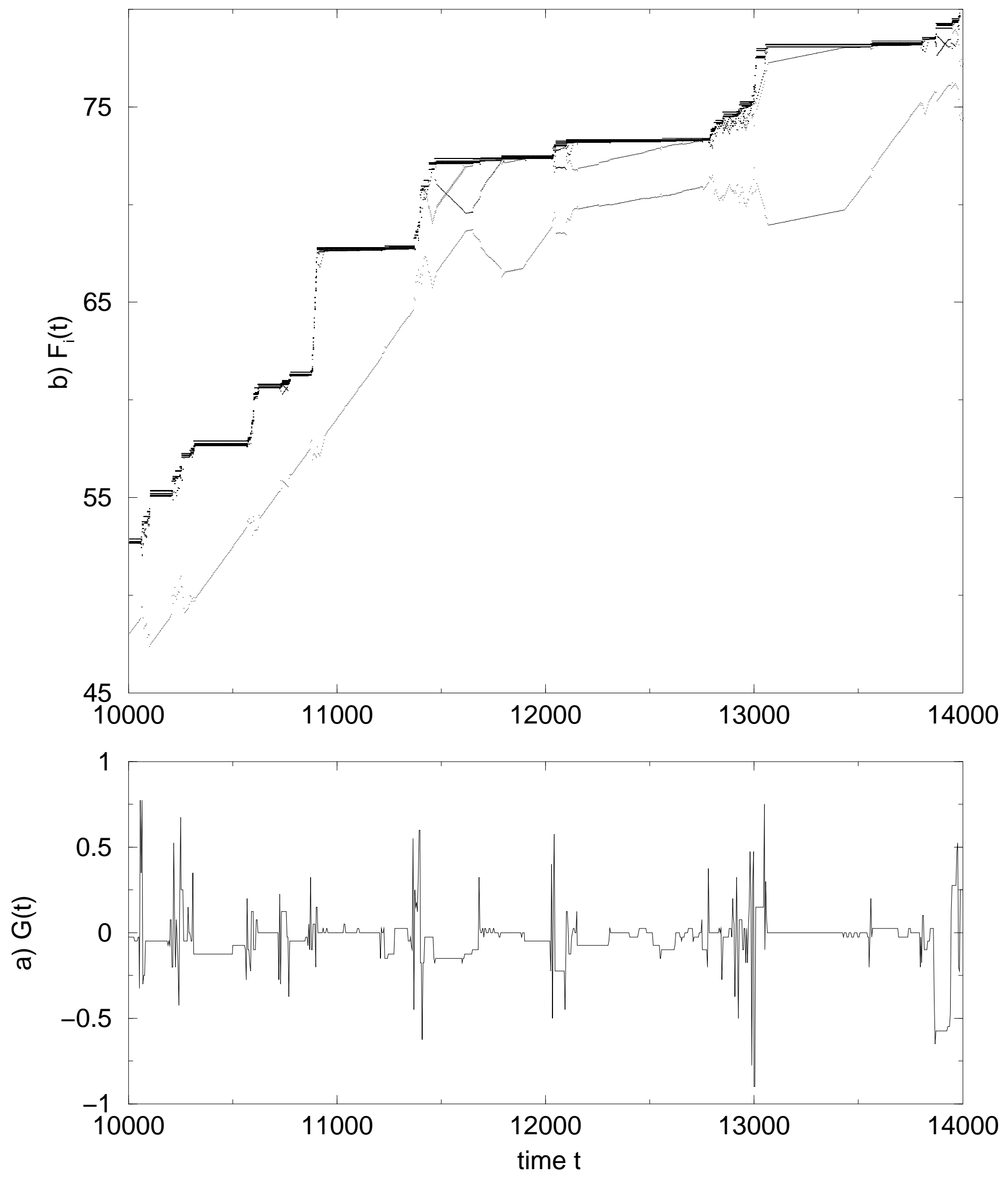

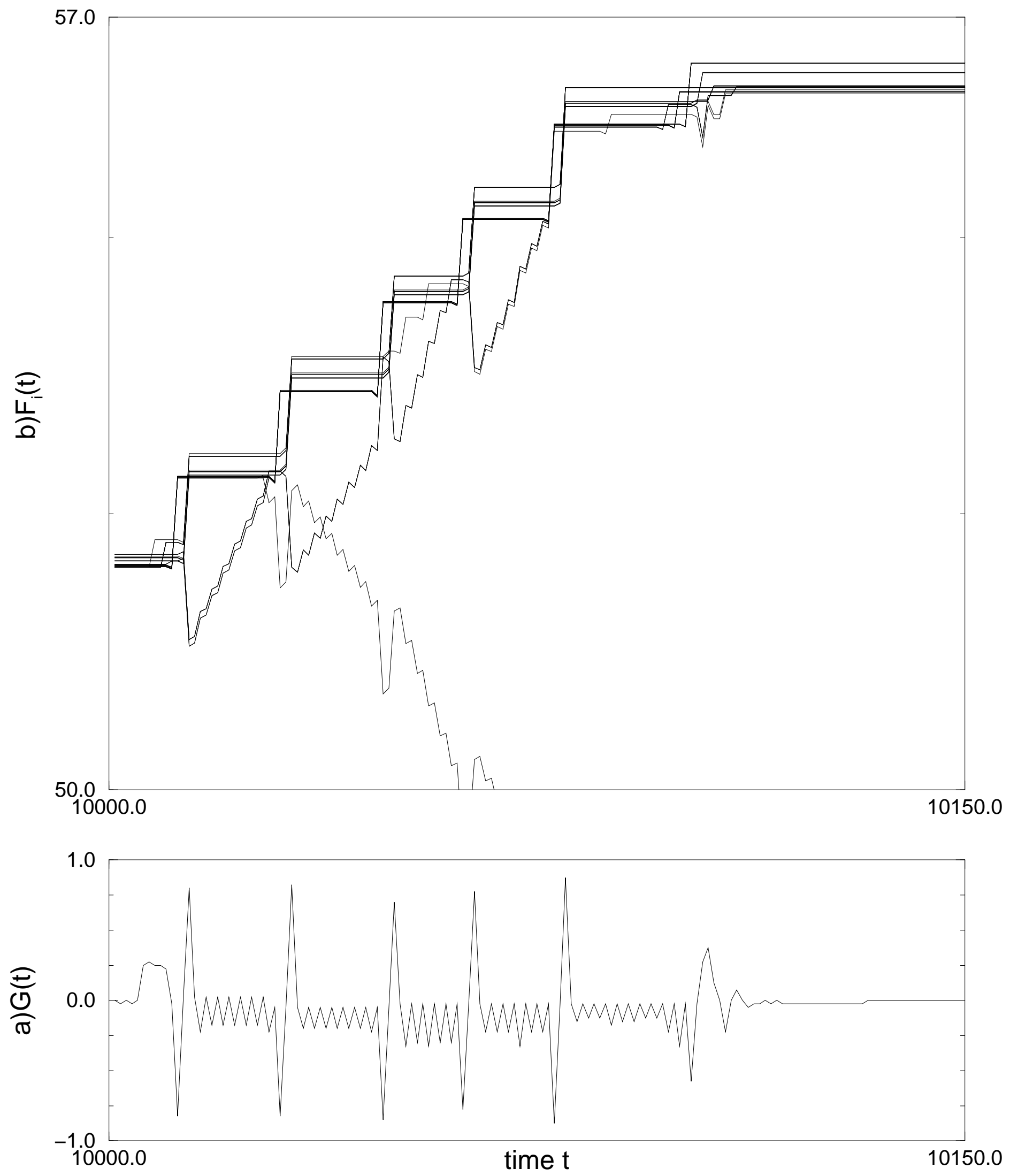

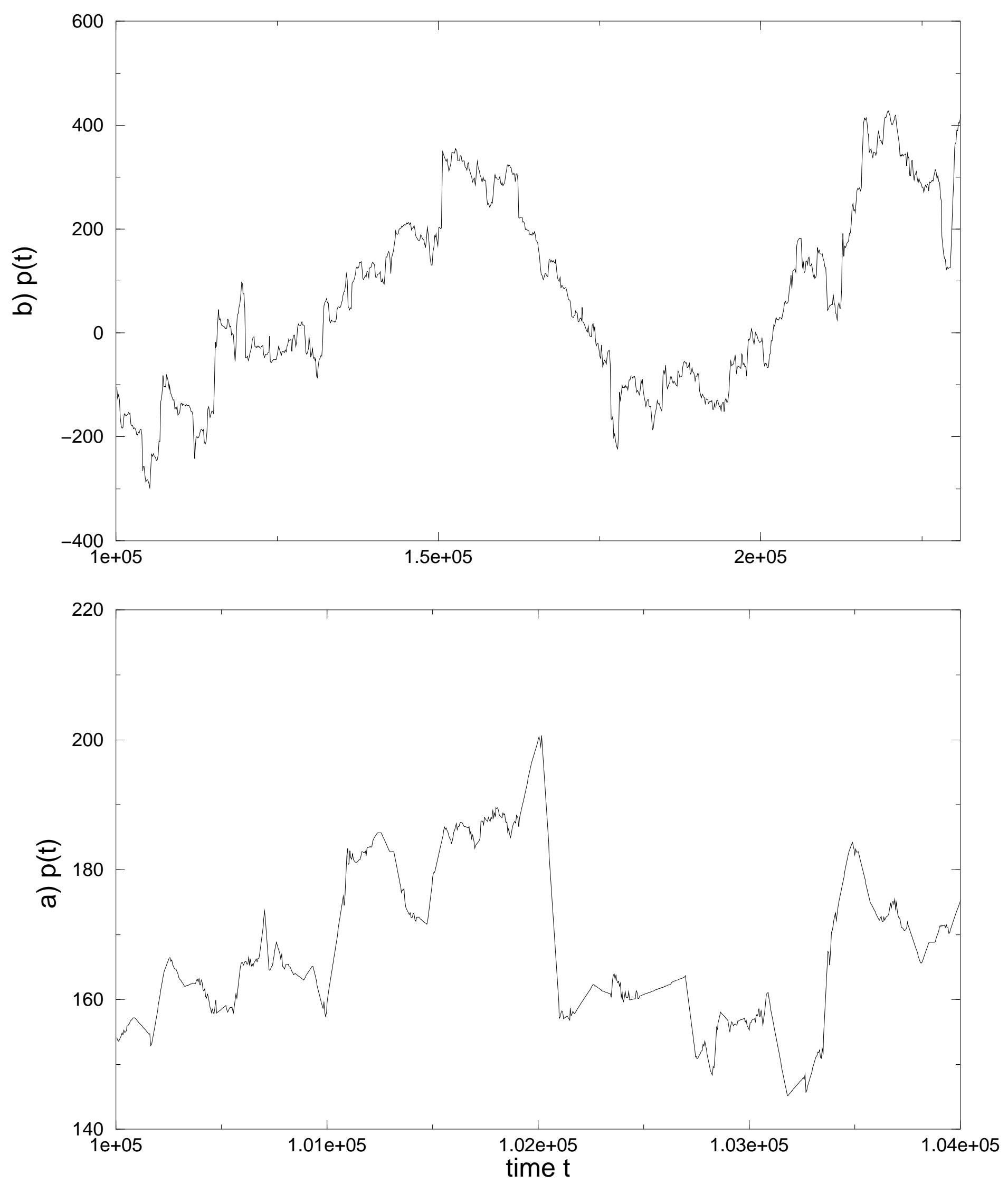

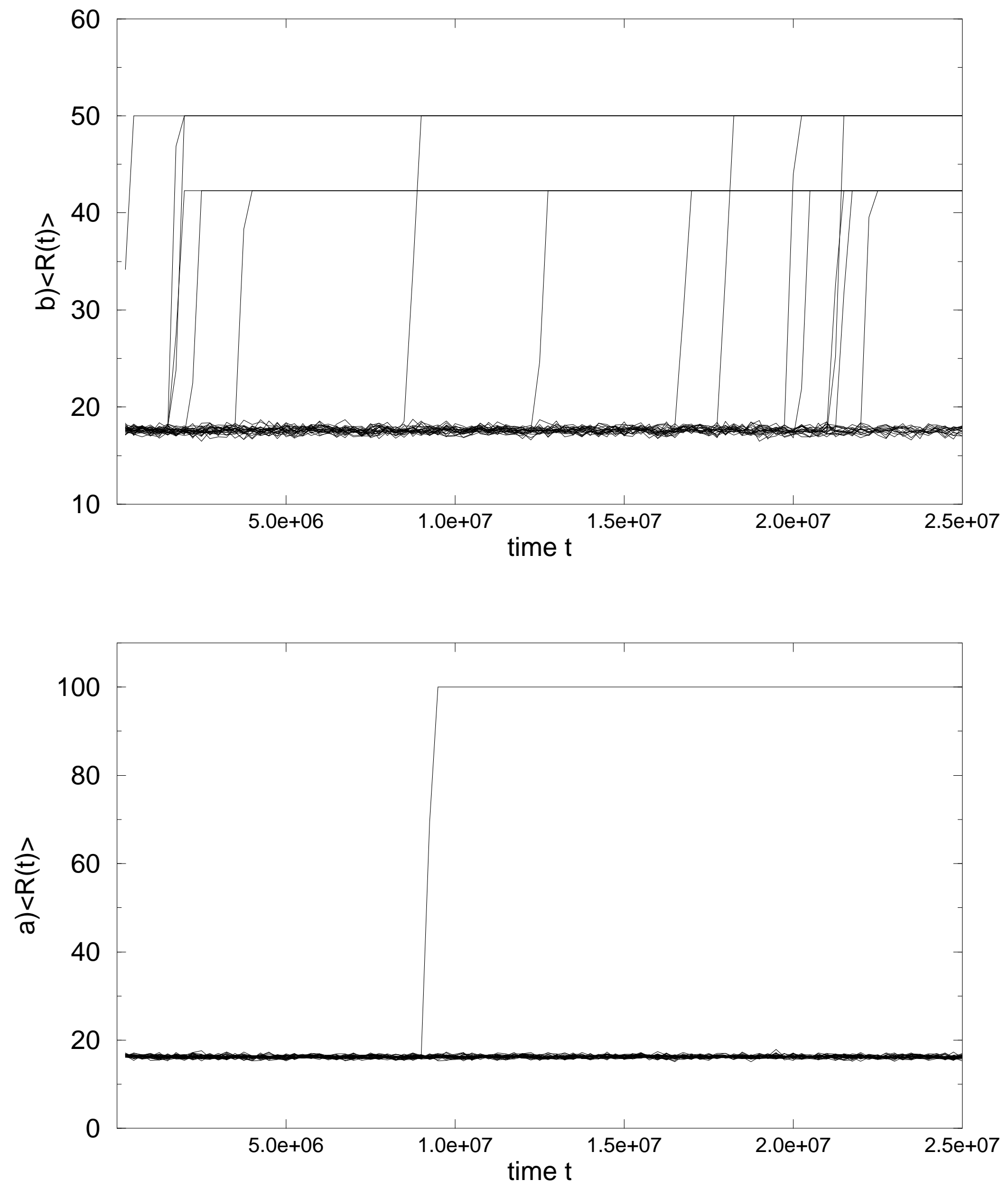

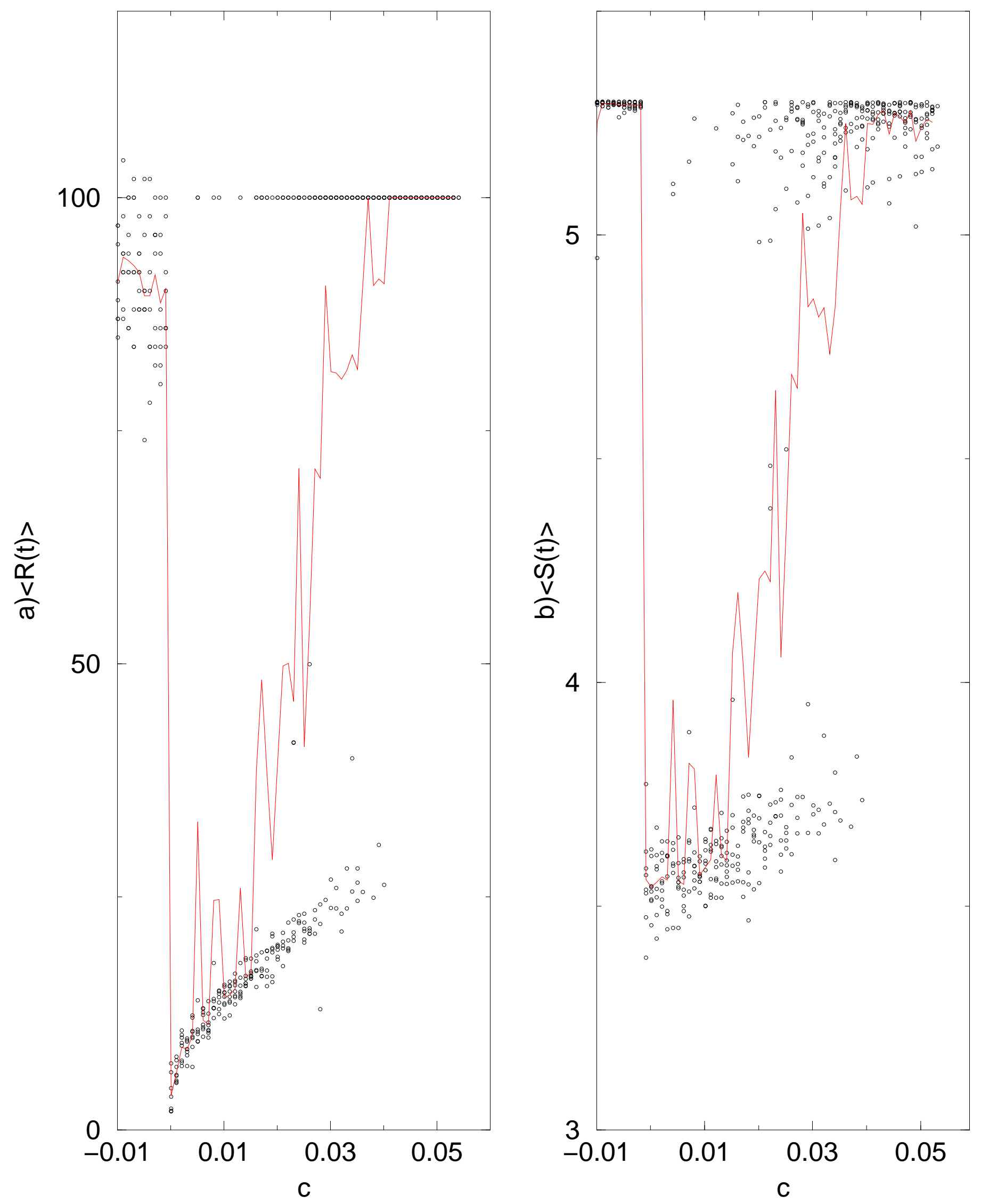


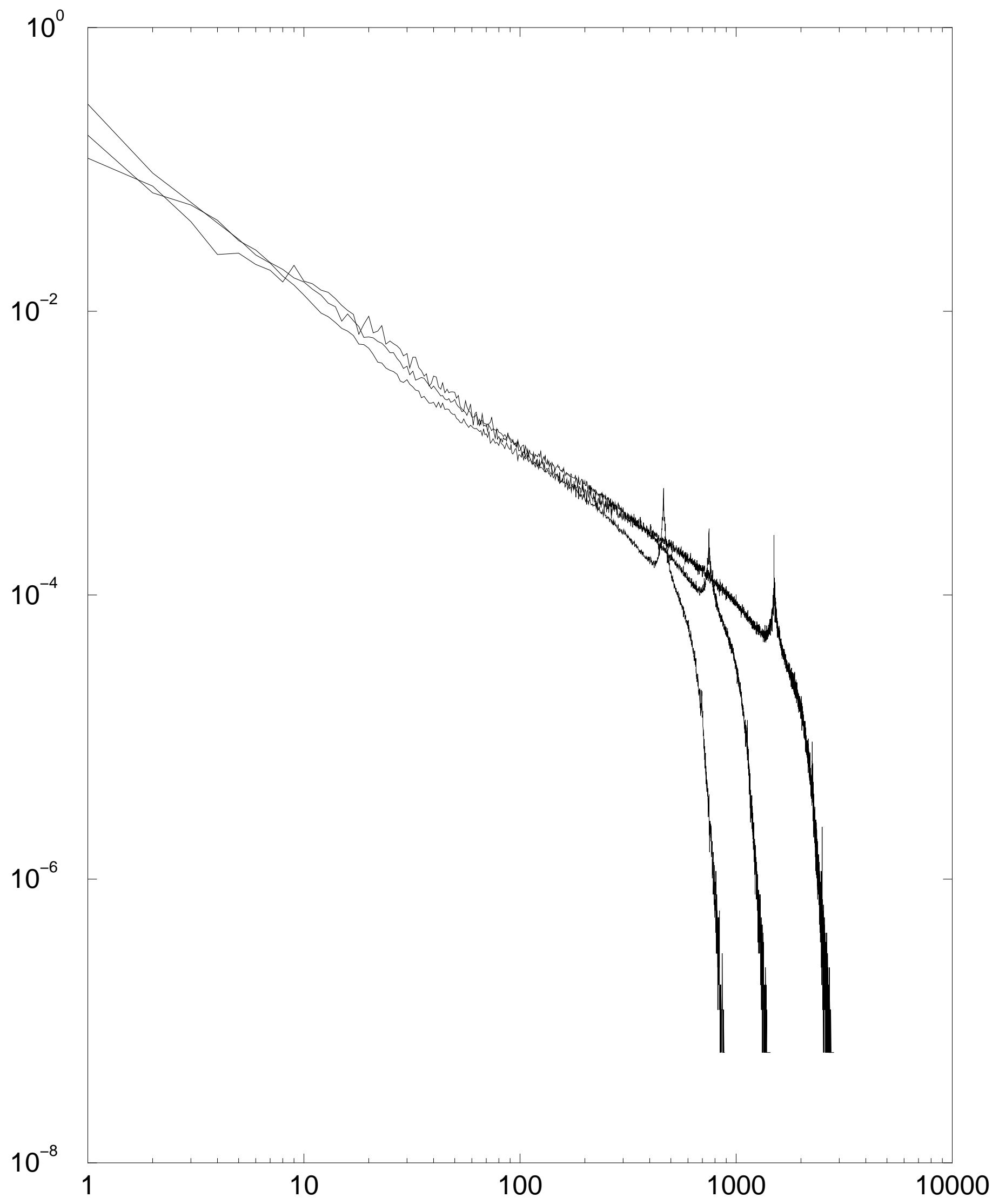



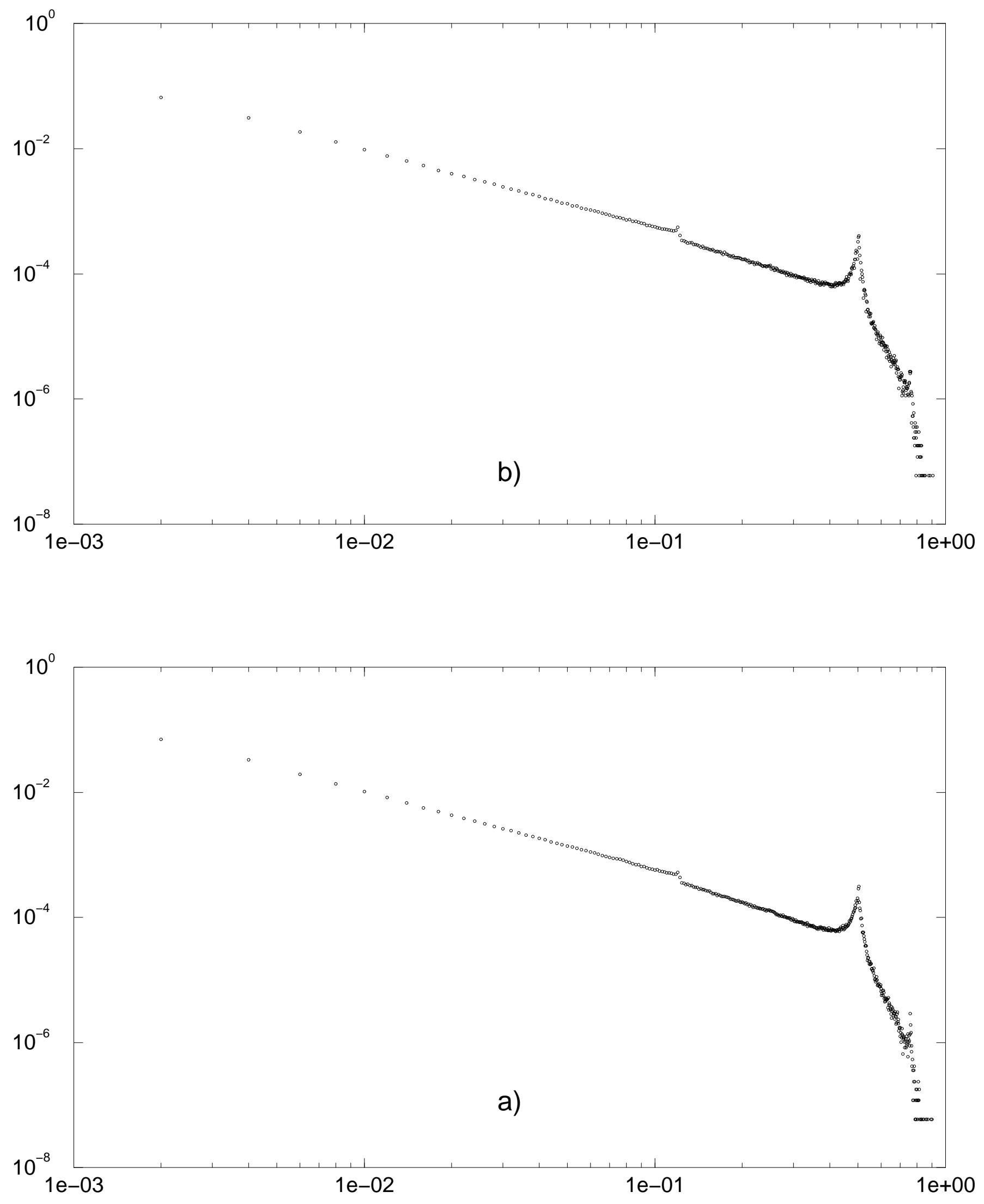


\section{Figure Captions}

Fig.1. a) Daily returns time series $G(t)$ showing intermittent high volatility. b) Corresponding fitnesses $F_{i}(t)$ against time $t$, showing periods of relative stasis punctuated by sudden increases in mean-fitness. The system size $N=80$ and $c=0.002$.

Fig.2. Detail from Fig.1. a) $G(t)$ b) $F_{i}(t)$ Partial synchronization persists for several time steps. Most of the fitnesses cluster into two groups and alternately topple, interleaved by the mean-fitness,(not shown). Not all the fitnesses $F_{i}(t)$ are shown.

Fig.3. Price $P(t)$ time series for $c=0.013$. a) 4096 time steps. b) $4096 \times 32$ time steps.

Fig.4. $<R(t)>$ against time $t$ for a) $c=0.0113, \mathrm{~b}) c=0.01$, averaging is over time $T=250,000$

Fig.5. a) $<R(t)>$ and $\mathrm{b})<S(t)>$ against parameter $c$. The line is an average of the 8 points.

Fig.6. Avalanche size probability distribution $P(R)$ against $R$, for size $N=923, N=$ 1500 , and $N=3000$. The cost $c=0.0113$.

Fig.7. Avalanche size probability distribution $P(\Delta F)$ against $\Delta F$, for size a) $N=1500$ and b) $N=923$. The cost $c=0.0113$. Separate scaling regimes are observed. 